\title{
FUNCTIONAL OUTCOMES AFTER PERCUTANEOUS NEPHROSTOMY IN HYDRONEPHROTIC KIDNEYS IN ADULT POPULATION
}

\author{
MD. MUAZZAM HOSSAN ${ }^{1}$, MD. FAZAL NASER ${ }^{2}$, MD. ROKONUZZAMAN KHAN ${ }^{3}$, MD AMIRUL ISLAM $^{4}$, \\ MD GOLAM KABIR ${ }^{5}$
}

\begin{abstract}
Objective: To evaluate the results of renal function after percutaneous nephrostomy in hydronephrotic kidneys in adult population.

Methods: This study was performed on 57 patients of more than 18 years of age with hydronephrosis due to PUJ obstruction and split renal function $<10 \%$ at the Department of Urology, Dhaka Medical College Hospital. Patients with concomitant pathologies like stone, pyonephrosis and VUJ obstruction were excluded from the study. Their evaluation included split renal function (SRF), glomerular filtration rate (GFR), specific gravity of urine draining through nephrostomy tube, volume of urine through nephrostomy tube. Statistical analysis was performed by SPSS version 13 and the test statistics used to analyse the data were descriptive statistics and Repeated measure ANOVA.

Results: Mean age of the patients was 33.4+13.0 years, mean SRF increased from $6.2 \%$ at baseline to $18.6 \%$ after 6 weeks of PCN $(p<0.001)$, mean GFR increased from $6.2 \mathrm{ml} / \mathrm{min} / 1.73 \mathrm{sq}$-meter at baseline to $18.6 \mathrm{ml} / \mathrm{min} / 1.73 \mathrm{sq}-\mathrm{meter}$ at the end of week 6, mean specific gravity of urine significantly increased from 1.009 at baseline to 1.019 after 6 weeks, volume of urine at $2^{\text {nd }}$ week was $316 \mathrm{ml} / 24$ hours and nearly $363 \mathrm{ml} 24$ hours after 6 weeks of PCN ( $p<0.001)$. GFR and SRF improved in $78.95 \%$ case

Conclusions: Most of the poorly functioning hydronephrotic kidneys showed functional improvement following PCN and hence not all such kidneys should be removed without subjecting them to a trial of $P C N$.
\end{abstract}

Key words: Hydronephrosis, SRF, GFR, PCN.

Bangladesh J. Urol. 2018; 21(2): 93-97

\section{Introduction:}

Hydronephrosis is a condition occurring due to blockage of urine flow, resulting in increased pressure within the collecting system leading to renal injury. It is a potentially life threatening condition and immediate measures are taken to decompress the kidneys.

1. Assistant Professor, Department of Urology, SSANSH, Khulna,

2. Associate Professor, Department of Urology, ShSMC, Dhaka,

3. Assistant Professor, Department of Urology, MA Rahim Medical College, Dinajpur,

4. Assistant Professor, Department of Urology, Sheikh Sayera Khtun Medical College, Gopalgong,

5. Assistant Professor, Department of Urology, Khulna Medical College, Khulna,

Correspondences: Dr. Md. Muazzam Hossan, Assistant Professor, Department of Urology, SSANSH, Khulna

Received: 9 August $2017 \quad$ Accepted: 05 March 2018
Congenital UPJ obstruction is the most common cause of hydronephrosis in children but not uncommon in adults. The management of hydronephrosis with poor renal function has been the subject of debate.Clinically some kidneys with $<10 \%$ split renal function become difficult to interpret whether corrective surgery or nephrectomy is needed ${ }^{1}$. In the presence of obstruction, poor or absent renal function on the intravenous urogram or DTPA renogram may not give a clear idea about the reasonable functional recovery of the kidney 2,3 . In this circumstance, percutaneous nephrostomy relieves obstruction and some time gives renal functional recovery in some cases. Thus, PCN can predict the outcome of definite surgery like pyeloplasty. Corrective surgery without a trial of percutaneous nephrostomy in poorly functioning hydronephrotic 
kidney may not recover renal function and demands second surgery (nephrectomy). Depending upon the improvement of renal function following PCN, definitive surgery can be done safely. Renal functional improvement following PCN in children is good but in adult there is some controversy. So we want to see the result in adults.

\section{Methods and Materials:}

This observational study was carried out at the Department of Urology, Dhaka Medical College Hospital during the period from July 2008 to June 2010.The cases were selected with the inclusion criteria having UPJ obstruction of either sex more than 18 years of age and split renal function $<10 \%$ (unilateral and / bilateral). Patients with concomitant pathologies like stone, pyonephrosis and VUJ obstruction were excluded from the study.

All patients were evaluated by detail history, clinical examination, and relevant investigations having similar protocol. Preoperative IVU was done in all patients to evaluate the condition of kidney. DTPA renogram was performed to assess the preoperative split renal function (SRF) and glomerular filtration rate (GFR) in all patients. The $\mathrm{PCN}$ was performed under ultrasound guidance after administering local anesthesia supplemented by intravenous sedation with pethidine $(0.5 \mathrm{mg} / \mathrm{kg})$ and promethazine $(0.25 \mathrm{mg} / \mathrm{kg})$.Urine sample was collected for specific gravity measurement and patients were discharged after few hours of observation, with the advice to take low-dose nitrofurantoin prophylaxis as long as the catheter remained in place. All 57 patients underwent PCN drainage followed-up at 2 weeks, 4 weeks and 6 weeks. During follow up serum creatinine, specific gravity and volume of urine collected through nephrostomy tube and renogram with SRF and GFR were determined.

\section{Results:}

Mean age of the patients was $33.4+13.0$ years (range18to70years).Clinical presentations were flank mass, flank pain, UTI and haematuria.

Table-I

Distribution of patients by clinical presentation $\left(n=57^{*}\right)$

\begin{tabular}{lcc}
\hline Clinical presentation & Frequency & Percentage \\
\hline Flank mass & 49 & 86.0 \\
Flank pain & 44 & 77.2 \\
UTI & 23 & 40.4 \\
Haematuria & 07 & 12.3 \\
\hline
\end{tabular}

\# Total will not correspond to $100 \%$ because of multiple responses.
Mean specific gravity of urine significantly increased from 1.009 at baseline to 1.019 after 6 weeks of PCN.

Table II

Gradual change in specific gravity of urine $(n=57)$

\begin{tabular}{|c|c|c|c|}
\hline \multirow[t]{2}{*}{ Time interval } & \multicolumn{2}{|c|}{ Specific gravity of urine } & \multirow[t]{2}{*}{ p-value } \\
\hline & Mean & SD & \\
\hline Baseline & 1.009 & 0.004 & \\
\hline 2 weeks & 1.016 & 0.005 & $<0.001$ \\
\hline 4 weeks & 1.018 & 0.005 & \\
\hline 6 weeks & 1.019 & 0.005 & \\
\hline
\end{tabular}

\# Repeated measure ANOVA statistics was employed to analyse the data. ; p refers to overall change from baseline to 6 weeks.

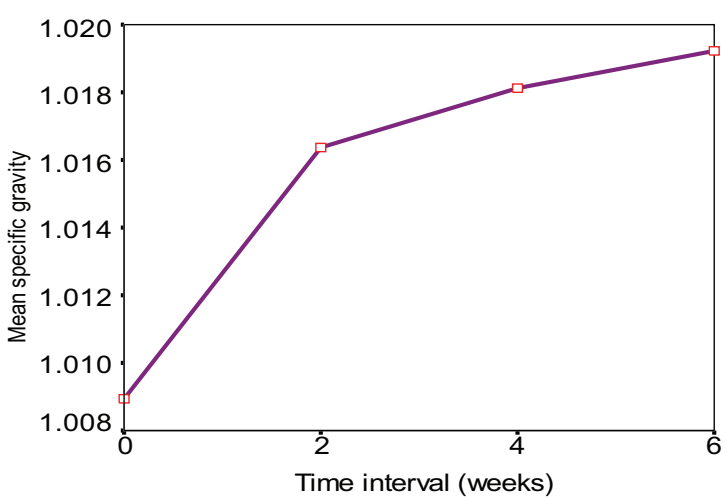

Fig.-1: Gradual change in specific gravity of urine $(n=$ 57)

Volume of urine at $2^{\text {nd }}$ week was $316 \mathrm{ml} / 24$ hours and nearly $363 \mathrm{ml} / 24$ hours after 6 weeks of PCN $(p<$ 0.001).

Table III

Gradual changes in urine volume from 2 to 6 weeks $(n=57)$

\begin{tabular}{llll}
\hline Time interval & \multicolumn{2}{c}{ Urine volume $(\mathrm{ml})$} & p-value \\
& Mean & SD & \\
\hline 2 weeks & 316.5 & 170.2 & \\
4 weeks & 335.1 & 193.1 & $<0.001$ \\
6 weeks & 363.7 & 204.2 & \\
\hline
\end{tabular}

\# Repeated measure ANOVA statistics was employed to analyse the data; $p$ refers to overall change from 2 to 6 weeks. 
Functional Outcomes after Percutaneous Nephrostomy In Hydronephrotic Kidneys In Adult Population

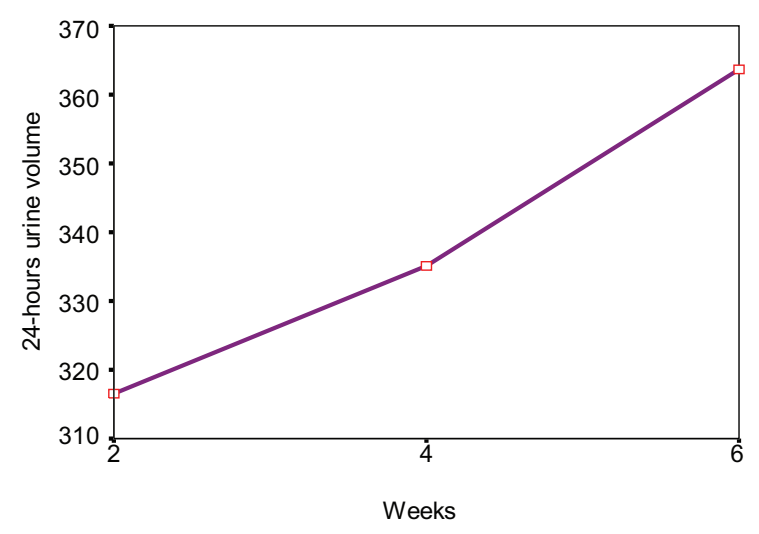

Fig.-2: Gradual changes in urine output $(n=57)$

Mean SRF increased from $6.2 \%$ at baseline to $18.6 \%$ after 6 weeks of PCN $(p<0.001)$.

Table IV

Change in SRF over time following PCN $(n=57)$

\begin{tabular}{lccc}
\hline Time interval & \multicolumn{2}{c}{ SRF (\%) } & p-value \\
& Mean & SD & \\
\hline Baseline & 6.2 & 2.1 & \\
2 weeks & 13.8 & 5.8 & $<0.001$ \\
4 weeks & 16.2 & 7.3 & \\
6 weeks & 18.6 & 8.3 & \\
\hline
\end{tabular}

\# Data were analysed using Repeated measure ANOVA; $p$ refers to overall change from baseline to 6 weeks.

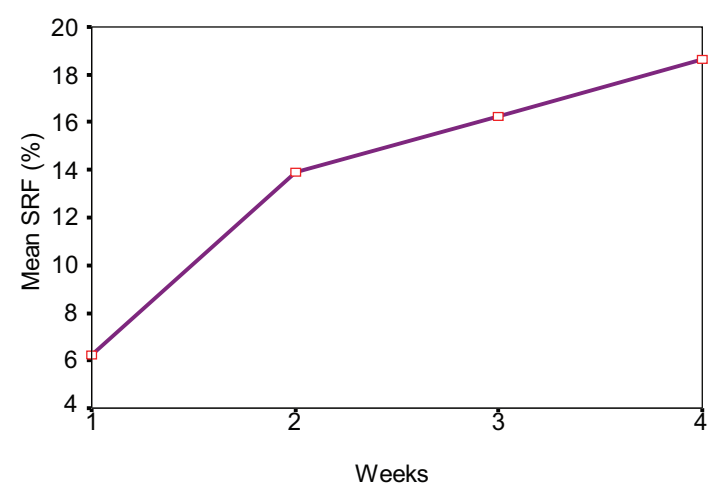

Fig.-3: Changes in split renal function following PCN $(n=57)$

Mean GFR increased from $6.2 \mathrm{ml} / \mathrm{min} / 1.73 \mathrm{sq}$-meter at baseline to $18.6 \mathrm{ml} / \mathrm{min} / 1.73 \mathrm{sq}-\mathrm{meter}$ at the end of week GFR and SRF improved in $78.95 \%$ case.
Table V

Change in GFR over time following PCN $(n=57)$

\begin{tabular}{lccl}
\hline Time interval & GFR (ml/min/sq-meter) & p-value \\
& Mean & SD & \\
\hline Baseline & 9.0 & 3.6 & \\
2 weeks & 17.4 & 7.3 & $<0.001$ \\
4 weeks & 19.9 & 8.8 & \\
6 weeks & 22.8 & 10.3 & \\
\hline
\end{tabular}

\# Data were analysed using Repeated measure ANOVA; $p$ refers to overall change from baseline to 6 weeks.

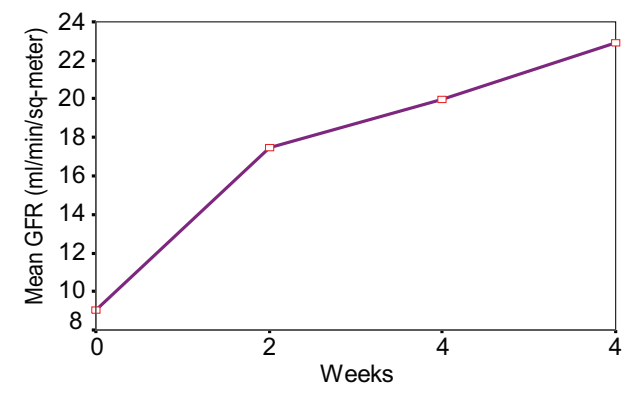

Fig. 4: Overall changes in GFR following PCN $(n=57)$

\section{Discussion:}

Hydronephrosis due to congenital UPJO is the most common cause of obstructive uropathy in children; however, it is also more frequent than generally appreciated in the adult population (Scardino et al., $1981)^{4}$. It occurs more in males. UPJO producing unilateral hydronephrosis predominantly involves the left kidney (Dubuisson et al., 1983; Heloury et al., $1986)^{5,6}$. In this study similar findings were found i.e., male preponderance $(75 \%)$ and predominant left-sided involvement $(73.7 \%)$. The outcome of the present study was evaluated in terms of volume of urine through nephrostomy tube, specific gravity of urine, changes in SRF and GFR following PCN. Of them SRF and GFR were the main outcome measures.

There is debate regarding the duration of $\mathrm{PCN}$ drainage for evaluation of renal function. Pode et al., (1982) reported functional recovery in 2 of 4 adult kidneys after PCN drainage and recommended a minimum of 4 weeks of drainage to assess recoverability. In another study the optimal period of drainage before maximal recovery was described as two months (Kerr, 1956) ${ }^{7}$. In the present study PCN drainage was maintained for 6 weeks. The present study demonstrated a modest 
increase in SRF from $6.2 \%$ at baseline to $18.6 \%$ at week 6 . SRF of $78.9 \%$ patients improved significantly during the period. Son et al., $(1996)^{8}$ reported significant improvement of SRF in $19(82.60 \%)$ out of 23 poorly functioning kidneys. Gupta et al., (2001) ${ }^{1}$ reported $70 \%$ of the poorly functioning kidneys to improve after a 4 weeks trial of PCN in children. Aziz et al., (2002) ${ }^{9}$ reported improvement of split renal function in all children with pre-PCN SRF $<10 \%$ after 6 weeks of PCN drainage. Ransley et al., (1990) ${ }^{10}$ recommended a trial of PCN drainage in kidneys with SRF less than $20 \%$ on initial renography. Dhillon, $(1998)^{11}$ recommended that kidneys with an initial SRF of less than $10 \%$ would not benefit from PCN drainage and hence should be spared from such trials. The results of the present study are not in conformity with this recommendation, because $78.9 \%$ (45 out of 57 ) of the poorly functioning UPJO kidneys (SRF $<10 \%)$ in adult exhibited improvement after PCN.

In the present study the mean GFR of poorly functioning kidneys was $9.0 \mathrm{ml} / \mathrm{min} / 1.73 \mathrm{sq}$-meter at baseline which increased to $22.8 \mathrm{ml} / \mathrm{min} / 1.73 \mathrm{sq}-$ meter at the end of week 6 . A preoperative GFR value of $10 \mathrm{~mL} / \mathrm{min} / 1.73$ $\mathrm{m}^{2}$ was estimated as the cut-off point that can determine the best prediction of stabilization or improvement of renal function after the relief of obstruction. However, this comment is dissimilar with the present study and that might be due to factors such as degree and duration of obstruction, greater compliance of collecting system and presence of pyelolymphatic backflow (Shokeir et al., 2002) ${ }^{12}$ may influence the recoverability of renal function after relief of obstruction. In this study urine output through the nephrostomy tube became normal within 6 weeks even with poor renal function (SRF $<10 \%$ ) which was consistent with the findings of Dubuisson et al., $(1983)^{5}$. Specific gravity of urine also increased during the 6 weeks period following percutaneous nephrostomy (PCN). In the presence of obstruction with poor function of kidney documented by renography, recoverability of useful renal function cannot reliably be predicted (Irving et al., 1987; O'Reilly, 1982) ${ }^{13,3}$ Factors influencing renal functional return after prolong periods of obstruction are degree and duration of obstruction, patient's age, greater compliance of collecting system and presence of pyelolymphatic backflow (Shokeir et al., 2002) ${ }^{12}$. The best evaluation of the capacity of a kidney to recover after obstruction is a period of therapeutic trial (Winfield et al., 1984) $)^{14}$ and PCN has been recommended for this purpose. From the present study it may be recommended that as the likelihood of recovery of a severely damaged kidney (SRF $<10 \%$ ) following PCN is fairly satisfactory and none of the adults with poorly functioning hydronephrotic kidney should undergo nephrectomy without subjecting them to a PCN trial.

Conclusion: PCN drainage for 6 weeks in patients with UPJO and an SRF of less than $10 \%$ aids in differentiating kidneys that are likely to recover from those that are unlikely to recover renal function. Majority of the patients with poorly functioning hydronephrotic kidneys showed improvement in function and hence not all such kidneys should be removed without subjecting them to a trial of PCN.

\section{Conflict of Interest: None Declared}

\section{References:}

1. Gupta DK, Chandrasekharam VVSS, Srinivas M and Bajpai M 2001, 'Percutaneous nephrostomy in children with ureteropelvic junction obstruction and poor renal function', Urol, vol. 57, pp.547-50.

2. Pode D, Shapiro A, Gordon R 1982, 'Percutaneous nephrostomy for assessment of functional recovery of obstructed kidneys', Urology, vol. 19, pp. 482-5.

3. O'Reilly $\mathrm{PH}$ 1982, 'Role of modern radiological investigations in obstructive uropathy', $\mathrm{Br}$ Med J (Clin Res Ed), vol. 284, pp. 1847-51.

4. Scardino PT, Scardino PL 1981, 'Obstruction at the ureteropelvic junction, in Bergman(Ed): The ureter, New York, Springer-Verlag, chap 33, pp 697-716.

5. Dubuisson RL, Eichelberger RP, and Jones TB 1983, 'A simple modification of real-time sector sonography to monitor percutaneous nephrostomy', Radiology, vol.146, pp. 232.

6. Heloury Y, Schmitt P, Allouch G et al. 1986, 'Treatment of neonatal hydronephrosis by malformation of the ureteropelvic junction: interest of percutaneous nephrostomy', Eur Urol, vol. 12, pp. 224-9.

7. Kerr WS Jr 1956, 'Effects of complete ureteral obstruction in dogs on kidney function', Am J Physiol, vol. 184, pp. 521.

8. Son H, Kim KM, Choi H 1996, 'Percutaneous Nephrostomy in infants and children.Korean', J Urol, vol. 27, no. 2, pp. 163-8. 
9. Aziz MA, Hossain AZ, Banus T, Karim MS, Islam $\mathrm{N}$, Sultana $\mathrm{H}$ et al. 2002, 'In hydronephrosis less than $10 \%$ kidney function is not an indication for nephrestomy in children', Eur J Pediatric Surg, vol. 12. pp. 302-7.

10. Ransley PG, Dhillon HK, Gordon I 1990, 'The postnatal management of hydronephrosis diagnosed by prenatal ultrasound, J Urol, vol. 144, pp. 584-7.

11. Dhillon HK 1998, 'Prenatally diagnosed hydronephrosis: The Great Ormond Street experience', Br J Urol, vol. 81, no. (suppl. 2), pp. 39-44.
12. Shokeir AA, Shoma AM, Abubieh EA, et al 2002, 'Recoverability of renal function after relief of acute complete ureteral obstruction: Clinical prospective study of the role of renal resistive index', Urology, vol. 59, pp.506-510.

13. Irving HC, Arthur RJ, and Thomas DF 1987, 'Percutaneous nephrostomy in paediatrics', Clin Radiol, vol. 38, pp. 245-8.

14. Winfield AC, Kirchner SG, Brun ME 1984, 'Percutaneous nephrostomy in neonates, infants, and children', Radiology, vol. 151, pp. 617-9. 